\title{
Comparisons of Mixed Systems Using Mean Vitality Function
}

\author{
A. Toomaj ${ }^{*}$ \\ Department of Statistics, Faculty of Mathematical Sciences, \\ Ferdowsi University of Mashhad, Mashhad, 91775-1159, Iran \\ ab.toomaj@gmail.com ${ }^{\dagger}$ \\ M. Doostparast \\ Department of Statistics, Faculty of Mathematical Sciences, \\ Ferdowsi University of Mashhad, Mashhad, 91775-1159, Iran \\ doustparast@um.ac.ir;doostparast@math.um.ac.ir
}

Received 15 March 2013

Accepted 21 December 2013

\begin{abstract}
The concept of system signature is widely used for comparing of mixed (coherent) systems. In practical situations, it is usually impossible to compare mixed systems. To do this, some partial orders are considered in the literature. In this paper, we define a new concept of stochastic order for comparing mixed systems based on mean vitality function. We assume that component lifetimes of competing systems are independent and identically distributed (iid) with a common distribution function. The obtained results are extended for comparing systems in which the component lifetimes are iid while the common component lifetime distributions are not identical. Some illustrative examples are also given.
\end{abstract}

Keywords: Coherent System; MRL; MVF; Mixed System; Stochastic Orders; Signature.

2000 Mathematics Subject Classification: 62N05, 94A17

\section{Introduction}

In engineering reliability, mean residual lifetime (MRL) of distribution is widely used in applications. This function measures the expected lifetime of an individual given that its survived up time $t$. More precisely, let $X$ be a random lifetime with cumulative distribution function (cdf) $F$ with a finite moment. The MRL is defined as

$$
m(t)=E(X-t \mid X>t)=\frac{\int_{t}^{\infty} \bar{F}(x)}{\bar{F}(t)} d x
$$

where $\bar{F}(t)=1-F(t)$ is the survival (reliability) function. If the cdf $F$ has the probability density function (pdf) $f$, then

$$
m(t)=v(t)-t
$$

\footnotetext{
*The corresponding author.

$\dagger$ Typeset author e-mail address in single line
} 
where $v(t)=E(X \mid X>t)=\frac{\int_{t}^{\infty} x f(x)}{\bar{F}(t)} d x$ is called vitality function (VF) or life expectancy; see, Kupka and Loo [10]. The functions VF and MRL play an important role in engineering reliability, biomedical sciences and survival analyzes; see Kotz and Shanbhag [9], Murari and Sujit [11], Ruiz and Navarro [17], Bairamov et al. [2] and the references therein. The notion of entropy as a measure of uncertainty, introduced by Shannon [21], has a fundamental importance in a wide variety areas such as probability and statistics, financial analyses, engineering and information theory; see e.g. Cover and Thomas [7]. For a continuous random variable $X$ with the $\operatorname{pdf} f$, the entropy of $X$ is defined as

$$
H(X):=-\int_{0}^{\infty} f(x) \log f(x) d x .
$$

Throughout this paper "log" stands for the natural logarithm. Recently, Rao et al. [15] introduced a new measure of information, called cumulative residual entropy (CRE) and used for measuring the residual uncertainty of a random variable. Specifically, for the random variable $X$ with the $\operatorname{cdf} F(x)$, the $C R E$ is

$$
\mathscr{E}(X):=-\int_{0}^{\infty} \bar{F}(x) \log \bar{F}(x) d x .
$$

This measure (1.4) in general is more stable than (1.3) since the distribution function is more regular and has a lot of mathematical properties. The CRE information has an applications in reliability engineering and computer vision; see, Rao [16] for more details.

A system is said to be coherent if every component of the system be relevant and the structure function of the system is monotone (see, e.g., Barlow and Proschan [5], p. 6). Comparison of coherent systems is an important issue in engineering reliability. A special case of coherent systems is the $k$-out-of- $n$ systems, which fails upon failure of the $k$-th component. Several papers are given for comparison purposes in literature such as Barlow and Proschan [5], Kochar et al. [8]. Recently, the signature of the system, introduced by Samaniego [18], facilitates comparison of systems. Let $T$ denote the lifetime of a coherent system consisting of $n$ independent and identically distributed (iid) components with lifetimes $X_{1}, \cdots, X_{n}$ which follow the common cdf $F$. Samaniego [18] showed that the reliability function of the system can be written as

$$
\bar{F}_{T}(t):=P(T>t)=\sum_{i=1}^{n} s_{i} \bar{F}_{i: n}(t), \forall t>0,
$$

where $X_{i: n}(i=1, \cdots, n)$ is the associated order statistics of $X_{1}, \cdots, X_{n}$ with the survival function

$$
\bar{F}_{i: n}(t)=\sum_{j=0}^{i-1}\left(\begin{array}{c}
n \\
j
\end{array}\right)[F(t)]^{j}[\bar{F}(t)]^{n-j}, 1 \leq i \leq n, t \geq 0 .
$$

From (1.5), one can see that the pdf of the lifetime $T$ is

$$
f_{T}(t)=\sum_{i=1}^{n} s_{i} f_{i: n}(t), \forall t>0
$$

where

$$
f_{i: n}(t)=\frac{n !}{(i-1) !(n-i) !}[F(t)]^{i-1}[\bar{F}(t)]^{n-i} f(t), 1 \leq i \leq n, t \geq 0 .
$$


The coefficients $s_{i}$ in (1.5) are called the signature of the system. More precisely, the system signature is the vector $\mathbf{s}=\left(s_{1}, \cdots, s_{n}\right)$ where $s_{i}=P\left(T=X_{i: n}\right)$, for $1 \leq i \leq n$, is the probability of the $i$-th failure causes the system failure. Notice that $s_{1}, \cdots, s_{n}$ are nonnegative real numbers and do not depends on the cdf $F(t)$ and satisfy $\sum_{i=1}^{n} s_{i}=1$. Boland and Samaniego [6] showed that the representation (1.5) also holds for mixed systems which are defined as stochastic mixtures of coherent systems. One can see that the survival function of a given mixed system is a mixture of $k$-out-of- $n$ systems with weights $s_{i}$; For more details see, Samaniego [19].

The CRE is particularly suitable to describe the information in problems related to ageing properties of reliability theory based on the mean residual life function. The studying of the properties of the CRE have been discussed in the literature; See for example, Asadi and Zohrevand [1], Navarro et al. [12], Baratpour [3] and Psarrakos and Navarro [14] and the references therein. In this paper, we introduced a new partially ordering for comparison of mixed systems. There exists cases in which comparisons of mixed (coherent) systems based on common partial ordering such as usual, hazard rate and likelihood ratio orders are impossible; See for example Toomaj and Doostparast [22]. Hence, non-comparability of systems turns out to be a limitation in the metric. Therefore, the new proposed order is introduced for comparison purposes in some practical cases. In the rest of this paper, main results are derived in Section 2. First, we present some definitions which is used in the sequel. The new concept of order is defined and used it for comparing of mixed systems based on the concept of system signature when lifetimes of components of both systems are iid in two identical and nonidentical cases. Some examples are also given.

\section{Main results}

In this section, we introduce the concept of mean vitality function (MVF) order and is used for comparing of mixed systems based on the signature of system. First, we provide some definitions from Shaked and Shanthikumar [20].

Definition 2.1. The random variable $X \sim F$ is said to be smaller than $Y \sim G$ in the usual stochastic order, denoted by $X \leq_{s t} Y$, if $\bar{F}(t) \leq \bar{G}(t) \forall t>0$ or equivalently $F^{-1}(u) \leq G^{-1}(u), 0 \leq u \leq 1$, where $F^{-1}(u)=\sup \{x: F(x) \leq u\}, \bar{F}(t)=1-F(t)$ and $\bar{G}(t)=1-G(t)$ for all $t>0$.

Definition 2.2. Let $X$ and $Y$ be two discrete random variables with the probability mass functions $\mathbf{p}_{1}=\left(p_{11}, \cdots, p_{1 n}\right)$ and $\mathbf{p}_{2}=\left(p_{21}, \cdots, p_{2 n}\right)$, respectively. It is said to be $\mathbf{p}_{1} \leq s t \mathbf{p}_{2}$ if $\sum_{j=i}^{n} p_{1 j} \leq \sum_{j=i}^{n} p_{2 j}$ for $i=1, \cdots, n$.

Now, we define the concept of MVF order and then use it to compare of mixed systems when component lifetimes of the system are iid. First, we need the following lemma.

Lemma 2.1. Let $X \sim F$ and $Y \sim G$ be independent random variables with means $E(X)$ and $E(Y)$. If $X \leq_{s t} Y$, then

$$
\mathscr{E}(X)+E(X) \leq \mathscr{E}(Y)+E(Y)
$$


Proof. Since $\int_{0}^{\infty} \bar{F}(t) \log \left(\frac{\bar{F}(t)}{\bar{G}(t)}\right)-(E(X)-E(Y)) \geq 0$ (Baratpour and Habibi Rad [4]), it implies that

$$
-\int_{0}^{\infty} \bar{F}(t) \log \bar{F}(t) d t+E(X) \leq-\int_{0}^{\infty} \bar{F}(t) \log \bar{G}(t) d t+E(Y) .
$$

By using the fact that $\bar{F}(t) \leq \bar{G}(t)$, for $t>0$, we have

$$
-\int_{0}^{\infty} \bar{F}(t) \log \bar{G}(t) d t+E(Y) \leq-\int_{0}^{\infty} \bar{G}(t) \log \bar{G}(t) d t+E(Y),
$$

and the desired result follows.

The mean lifetime of the system based on the (1.5) is

$$
\mu_{T}=E(T)=\sum_{i=1}^{n} s_{i} \mu_{i: n},
$$

where $\mu_{i: n}$ for $i=1, \cdots, n$ stands for the expected lifetimes of the order statistics. From Lemma 2.1, we have

Theorem 2.1. Let $T$ be the lifetime of the mixed system with signature $s=\left(s_{1}, \cdots, s_{n}\right)$ consisting $n$ iid component lifetimes $X_{1}, \cdots, X_{n}$ coming from the $c d f F$. Then

$$
\mathscr{E}\left(X_{1: n}\right)+\mu_{1: n} \leq \mathscr{E}(T)+\mu_{T} \leq \mathscr{E}\left(X_{n: n}\right)+\mu_{n: n},
$$

where $T_{1: n}$ and $T_{n: n}$ stand for the lifetimes of the series and parallel systems, respectively.

Proof. From (1.5), we have $X_{1: n} \leq_{s t} T \leq_{s t} X_{n: n}$ and hence Lemma 2.1 completes the proof.

Theorem 2.1 says that the sum of the CRE and the mean lifetime of any arbitrary mixed system located between the sum of the CRE and mean lifetime of the series and parallel systems. Asadi and Zohrevand [1] proved $\mathscr{E}(X)=E(m(X))$. Therefore, from (1.2) the MVF of a random variable $X$ with finite mean $\mu=E(X)$ is

$$
\begin{aligned}
E(v(X)) & =E(m(X))+E(X) \\
& =\mathscr{E}(X)+\mu .
\end{aligned}
$$

Therefore, we have the following corollary.

Corollary 2.1. Under the conditions of Theorem 2.1, we have

$$
E\left(v\left(X_{1: n}\right)\right) \leq E(v(T)) \leq E\left(v\left(X_{n: n}\right)\right) .
$$

Corollary 2.1 says that the MVF of mixed systems are between the MVF's of the series and parallel systems. Hence, Expression (2.5) motivates the comparison of mixed systems based on MVF measure. Now, we introduce the concept of MVF order as follow:

Definition 2.3. Let $X$ and $Y$ be random variables with MVF's $E(v(X))$ and $E(v(Y))$, respectively. Then $X$ is said to be smaller than $Y$ in the MVF order, denoted by $X \leq_{m v f} Y$, if $E(v(X)) \leq E(v(Y))$. 
The MVF of the random variable $X$ with the cdf and the pdf $F$ and $f$, respectively, can be written as

$$
\begin{aligned}
E(v(X)) & =\int_{0}^{\infty} f(t) \frac{\int_{t}^{\infty} x f(x) d x}{\bar{F}(t)} d t \\
& =\int_{0}^{\infty} x f(x) \int_{0}^{x} h(t) d t d x \\
& =-\int_{0}^{\infty} x f(x) \log \bar{F}(x) d x .
\end{aligned}
$$

The last equality in (2.6) obtained by the fact that $\int_{0}^{x} h(t) d t=-\log \bar{F}(x)$. Let $T$ be the lifetime of the mixed system with $n$ iid component lifetimes coming from the cdf $F$ and signature s. The corresponding transformations of system's component lifetimes are iid random variables $U_{i}=F\left(X_{i}\right), i=1, \cdots, n$, which are uniformly distributed. It is known that the distribution of order statistics $W_{i}=F\left(X_{i: n}\right)$ is $\operatorname{Beta}(i, n-i+1)$ with the pdf

$$
g_{i: n}(w)=\frac{\Gamma(i) \Gamma(n-i+1)}{\Gamma(n+1)} w^{i-1}(1-w)^{n-i}, \quad 0<w<1,
$$

where $\Gamma($.$) stands for the complete gamma function. Also, the pdf and the survival function of the$ transformation $W=F(T)$ are $g_{W}(w)=\sum_{i=1}^{n} s_{i} g_{i: n}(w)$ and $\bar{G}_{W}(w)=\sum_{i=1}^{n} s_{i} \bar{G}_{i: n}(w)$, respectively, where the Jacobian of transformation for $T=F^{-1}(W)$ is $f(T)$. From (2.6) and the earlier transformations, we have

$$
\begin{aligned}
E(v(T)) & =-\int_{0}^{\infty} x f_{T}(x) \log \bar{F}_{T}(x) d x \\
& =-\int_{0}^{1} F^{-1}(u) g_{W}(w) \log \bar{G}_{W}(w) d w .
\end{aligned}
$$

Expression (2.8) can be easily evaluated by using some mathematical packages such as MATLAB and MAPLE. In the next example, we illustrate the comparison of the mixed systems using MVF by direct evaluating of the Expression (2.8) using MAPLE software version 12.

Example 1. Let $T_{1}$ and $T_{2}$ be lifetimes of two mixed systems with signatures $\mathbf{s}_{1}=\left(0, \frac{3}{7}, \frac{4}{7}\right)$ and $\mathbf{s}_{2}=\left(0, \frac{3}{8}, \frac{5}{8}\right)$, respectively, having 3 iid component lifetimes coming from the standard exponential distribution. It is easy to verify that $E\left(v\left(T_{1}\right)\right)=2.48$ and $E\left(v\left(T_{2}\right)\right)=2.55$ and hence $T_{1} \leq_{m v f} T_{2}$.

From Lemma 2.1 and (2.4), we have the following corollary.

Corollary 2.2. Let $X$ and $Y$ be independent lifetime random variable. If $X \leq_{s t} Y$, then $X \leq_{m v f} Y$.

Proposition 2.1. Let $T_{1}$ and $T_{2}$ be the lifetime of two mixed systems consisting of $n$ iid component lifetimes from the common cdf $F$ with signatures $\boldsymbol{s}_{1}$ and $\boldsymbol{s}_{2}$, respectively. If $\boldsymbol{s}_{1} \leq_{s t} \boldsymbol{s}_{2}$, then $T_{1} \leq_{m v f} T_{2}$.

Proof. Since $\mathbf{s}_{1} \leq_{s t} \mathbf{s}_{2}$, Theorem 1.2 duo to Navarro et al. [13] implies that $T_{1} \leq_{s t} T_{2}$ and then Corollary 2.2 completes the proof.

Now suppose that the component lifetimes of both systems are different. We have the following proposition.

Proposition 2.2. Let $T_{1}$ and $T_{2}$ be the lifetime of two mixed systems consisting of $n$ iid component lifetimes from the cdf's $F$ and $G$ with signatures $\boldsymbol{s}_{1}$ and $\boldsymbol{s}_{2}$, respectively. If $\boldsymbol{s}_{1} \leq_{s t} \boldsymbol{s}_{2}$ and $X \leq_{s t} Y$, then $T_{1} \leq_{m v f} T_{2}$. 
Proof. Since $\mathbf{s}_{1} \leq_{s t} \mathbf{s}_{2}$, Proposition 2.1 implies that

$$
\begin{aligned}
E\left(v\left(T_{1}\right)\right) & =-\int_{0}^{1} F^{-1}(u) f_{W_{1}}(u) \log \bar{F}_{W_{1}}(u) d u \\
& \leq-\int_{0}^{1} F^{-1}(u) f_{W_{2}}(u) \log \bar{F}_{W_{2}}(u) d u \\
& \leq-\int_{0}^{1} G^{-1}(u) f_{W_{2}}(u) \log \bar{F}_{W_{2}}(u) d u \\
& =E\left(v\left(T_{2}\right)\right) .
\end{aligned}
$$

The last inequality in (2.9) is obtained from $X \leq_{s t} Y$.

Example 2. Let $\mathbf{s}_{1}=\left(\frac{1}{4}, \frac{1}{4}, \frac{1}{2}, 0\right)$ and $\mathbf{s}_{2}=\left(0,0, \frac{1}{4}, \frac{3}{4}\right)$ be signatures of two systems consisting $n=4$ iid components with the common cdf $F$. Let $T_{1}$ and $T_{2}$ be the corresponding lifetimes of the systems. It is easy to verify that $\mathbf{s}_{1} \leq_{s t} \mathbf{s}_{2}$. Then Proposition 2.1 implies that $T_{1} \leq_{m v f} T_{2}$.

\section{Acknowledgments}

The authors are grateful to the Editor-in-Chief and anonymous referees for carefull reading of the manuscript.

\section{References}

[1] M. Asadi and Y. Zohrevand, On the dynamic cumulative residual entropy, J. Stat. Plan. Inference 137 (2007), 1931-1941.

[2] I. Bairamov, M. Ahsanullah and I. Akhundov, A residual life function of a system having parallel or series structures, Journal of Statistical Theory and Applications. vol. 1, no. 2 (2002) 119-132.

[3] S. Baratpour, Characterizations based on cumulative residual entropy of first-order statistics, Commun. Stat. Theory and Methods. 39 (2010) 3645-3651.

[4] S. Baratpour and A. Habibi Rad, Testing goodness-of-fit for exponential distribution based on cumulative residual entropy, Commun. Statist. Theo. Meth. 41 (2012), 1387-1396.

[5] R. E. Barlow and F. Proschan, Statistical Theory of Reliability and Life testing, (Silver springer, 1981).

[6] P. J. Boland and F. J. Samaniego The signature of a coherent system and its applications in reliability, Mathematical Reliability: An Expository Perspective, R. Soyer, T. Mazzuchi, and N.D. Singpurwalla (Editors), Kluwer Publishers, Boston (2004) 1-29.

[7] T. A. Cover and J. A. Thomas, Elements of Information Theory, (New Jersey: Wiley and Sons, Inc, 2006).

[8] S. C. Kochar, H. Mukerjee and F. J. Samaniego The signature of a coherent system and its application to comparisons among systems, Naval Res. Log. 46 (1999) 507-523.

[9] S. Kotz and D. Shanbhag, Some new approaches to probability distributions, Adv. Appl. Probab. vol 12 (1980) 903-921.

[10] J. Kupka and S. Loo, The hazard and vitality measures of aging, J. Appl. Prob. vol 26 (1989) 532-542.

[11] M. Murari and K. Sujit, Change point estimation in non-monotonic aging models, Ann. Inst. Statist. Math. 3 (1995) 483-491.

[12] J. Navarro, Y. del Aguila and M. Asadi, Some new results on the cumulative residual entropy, J. Stat. Plan. Inference. 140 (2010) 310-322.

[13] Navarro, J., Samaniego, F. J., Balakrishnan, N. and Bhattacharya, D. (2008). On the application and extension of system signatures to problems in engineering reliability, Naval Res. Log. 55, 313-327.

[14] G. Psarrakos and J. Navarro, Generalized cumulative residual entropy and record values, Metrika (2012). 
[15] M. Rao, Y. Chen, B. Vemuri and W. Fei Cumulative residual entropy: a new measure of information, IEEE Trans. Inf. Theory, $\mathbf{5 0}$ (2004).

[16] M. Rao, More on a new concept of entropy and information, J. Theoret. Probab. 18 (2005) 967-981.

[17] J. M. Ruiz and J. Navarro, Characterization of distributions by relationships between the failure rate and the mean residual life, IEEE Tran. Reliability. 43 (1994) 640-644.

[18] F. J. Samaniego, On closure of the IFR class under formation of coherent systems, IEEE Trans. Reliabil. (1985) 69-72.

[19] F. J. Samaniego, System Signatures and their Applications in Engineering Reliability, (Springer Science+Business Media, LLC, New York, 2007).

[20] M. Shaked and J. G. Shanthikumar, Stochastic Orders and their Applications, (Academic Press, San Diego, 2006).

[21] C. E. Shannon, A mathematical theory of communication, Bell System Tech. J. 27 (1948) 379-423 and 623-656.

[22] Toomaj, A. and Doostparast, M. (2013) Comparison of engineering systems with cumulative residual inaccuracy: A signature-based approach. Submitted. 